\section{Inanspruchnahme von Physio- und Ergotherapie bei Systemischer Sklerose}

\author{
Becetti K et al. Physical or occupational therapy \\ use in systemic sclerosis: a scleroderma \\ patient-centered intervention network cohort \\ study. J Rheumatol 2019; 46: 1605-1613
}

Physiotherapie und Ergotherapie können muskuloskelettale Beschwerden bei systemischer Sklerose verbessern. Dennoch nehmen vergleichsweise wenige Patienten diese Therapien in Anspruch. Eine neue Studie untersucht die Faktoren, die eine Inanspruchnahme der Therapien beeinflussen.

In der Studie sollten demografische, medizinische und psychologische Faktoren identifiziert werden, die mit der Inanspruchnahme von Physio- und Ergotherapie bei systemischer Sklerose assoziiert sind.

Die Wissenschaftler bestimmten die Rate und Indikation der Inanspruchnahme von Physio- bzw. Ergotherapie innerhalb der letzten 3 Monate vor Einschluss in die Studie. Die Probanden stammten aus der internationalen Scleroderma Patient-centered Intervention Network (SPIN) Kohorte. In die Kohorte wurden volljährige Patienten mit systemischer Sklerose nach den ACRKriterien von 2013 aus mehr als 40 Zentren aus Kanada, den USA und Europa eingeschlossen.
Die untersuchten demografischen Variablen waren u. a. Alter, Geschlecht, Ethnizität, Ausbildungsjahre, Beruf und Wohnort. Die medizinischen u. a. der Subtyp der systemischen Sklerose, die Anwesenheit des Raynaud-Phänomens, der modified Rodnan skin score (mRSS), digitale Ulcera, Gelenkkontrakturen, das Vorhandensein von Überschneidungssyndromen und eine kardiopulmonale Beteiligung. Um die körperliche Beeinträchtigung bestimmten zu können, sollten die Patienten den Scleroderma Health Assessment Questionnaire (SHAQ) und den Cochin Hand Function Scale (CHFS-II) beantworten. Um Symptome einer Depression bestimmten zu können, sollten die Patienten den Patient Health Questionnaire (PHQ-8) ausfüllen. Für die Bewertung anderer psychologischer Symptome auch die Patient Reported Outcomes Measurement Information System (PROMIS-29), Satisfaction with Appearance (SWAP), Social interaction anxiety scale-6 und Self-Efficacy for Managing Chronic Disease (SEMCD) Fragebögen.

In die Analyse wurden insgesamt $1627 \mathrm{~Pa}$ tienten eingeschlossen. $43 \%$ stammten aus den USA, 27\% aus Kanada, 17\% aus Frankreich, $10 \%$ aus dem Vereinigten Königreich und $2 \%$ aus Spanien. Das mittlere Alter der Patienten lag bei 54,9 $\pm 12,5$ Jahren. $87 \%$ der Patienten waren weiblich und $41 \%$ litten an einer diffusen systemischen Sklerose. Die mittlere Krankheitsdauer lag bei $11,4 \pm 8,8$ Jahren. Der mittlere mRSS lag bei $7,8 \pm 8,4$. Eine interstitielle Lungenerkrankung lag bei $36 \%$, ein pulmonaler arterieller Bluthochdruck bei $10 \%$ der Probanden vor.

Die Auswertung ergab, dass $23 \%$ der Patienten innerhalb der letzten 3 Monate Physio- oder Ergotherapie genutzt hatten, im Durchschnitt 9,8 \pm 10,7 Mal. Am häufigsten wurde die Therapie für eine Behandlung der Hände in Anspruch genommen (59\%). Die Inanspruchnahme war mit einer höheren Bildung (OR 1,08; 95 \% KI 1,04-1,12), dem Vorhandensein von mäßig schweren Kontrakturen kleiner Gelenke (OR 2,09; 95 \% KI $1,45-3,03)$ sowie von schweren Kontrakturen großer Gelenke (OR 2,33; $95 \%$ KI 1,144,74 ), weniger digitalen Ulzera (OR 0,70; $95 \%$ KI 0,51-0,95), einer höheren körperlicher Beeinträchtigung (OR 1,54; 95 \% KI 1,18-2,02) und höheren Schmerz-Scores 
(OR 1,04; $95 \%$ KI 1,02-1,06) assoziiert. Am häufigsten nutzen Patienten aus Frankreich (Rate $43 \%$ ) die Therapien, am seltensten die aus den USA (Rate $17 \%$ ). Insgesamt nutzte weniger als einer von 4 Patienten die Therapien.

\section{FAZIT}

Weniger als einer von 4 Patienten mit systemischer Sklerose nimmt eine Physio- oder Ergotherapie in Anspruch, obwohl diese Therapien die Beschwerden verbessern können. Patienten mit stärkeren muskuloskelettalen Beschwerden und stärkeren Schmerzen nehmen die Therapien eher in Anspruch. Die Autoren halten es für notwendig, die Zugänglichkeit zu Physio- und Ergotherapie zu erhöhen, etwa auch durch häuslicheund Online-Angebote.

Marisa Kurz M. Sc. B. A., München 